https://doi.org/10.31713/m919

\title{
THE RESEARCH OF INERTIAL CONVEYOR TRANSI- TIONAL CHUTE OSCILLATIONS INFLUENCE ON ITS TECHNICAL AND ECONOMIC INDICATORS
}

\author{
Serilko L. S. \\ National University of Water and Environmental Engineering, Ass. \\ Prof., PhD of Technical Sciences, Associate Professor of Theoretical \\ Mechanics, Engineering Graphics and Mechanical Engineering the \\ Department, Rivne, Ukraine

\section{Lyashuk O. L.} \\ Ternopil Ivan Puluj National Technical University, Professor, Doctor \\ of Technical Sciences, Head of Automobile Department, Ternopil, \\ Ukraine
}

\section{Sasyuk Z. K.}

National University of Water and Environmental Engineering, Ass. Prof., PhD of Agricultural Sciences, Associate Professor of Theoretical Mechanics, Engineering Graphics and Mechanical Engineering the Department, Rivne, Ukraine

\section{Serilko D. L.}

National University of Water and Environmental Engineering, Ass. Prof., PhD of Technical Sciences, Associate Professor of Construction, Road, Reclamation, Agricultural Machines and Equipment Department, Rivne, Ukraine.

\footnotetext{
Abstarct

The construction of an inertial conveyor for bulk materials transportation has been elaborated in order to increase productivity and reduce the energy intensity of the bulk materials transportation process. The inertial conveyor consists of a chute, which performs reciprocating motion on a fixed basis and actuating the reciprocating movement of the chute. The chute can perform not only a reciprocating motion, but also a movement in a direction that is perpendicular to the axis of the conveyor in a
} 
horizontal plane. It has been proposed to reduce the friction force between the load and the chute at a time when the speed of the chute relative to the load will be directed in the opposite direction. The analyses of known methods of solving nonlinear differential equations, which describe the motion of mechanical systems in the presence of dry friction has been done. A mathematical model of a bulk material particles motion over a surface that performs a harmonic motion has been elaborated. A construction of efficient inertial conveyor has been created. The influence patterns of structural and kinematic parameters of the inertial conveyor on the indicators of bulk materials transportation process by this vehicle have been experimentally investigated. A mathematical model of the flow of a bulk material in a rectangular chute that performs high intensity transverse oscillations in the plane of greatest inclination has been developed. It has been established experimentally that, in the presence of such oscillations, the material retains the shape of the chute and therefore its motion can be regarded as the motion of a solid on an inclined surface. The averaging method obtained an approximate solution of the equations of this motion, which satisfactorily agrees with the results of experimental studies.

The obtained dependences of the average velocity of the bulk material on the surface of the chute from the angle of inclination to the horizon and the coefficient of friction of the material on this surface can be applied in engineering calculations for solving specific practical problems.

Keywords: dynamic system, vibration transportation, inertial conveyor, transportation of bulk materials, transverse oscillations, friction force, vibration, oscillation frequency.

\section{Introduction}

A continuous improvement of the technical level of new mechanisms and machines is a characteristic feature of the modern development of world mechanical engineering.

The improvement of the constructions of various conveyors (belt, screw, inertial, etc.) is one of the directions of bulk materials transportation means development, which provides high productivity, technical and economic efficiency. The conducted analysis of designs of various vehicles constructions shows that in mining enterprises, for the transportation of bulk materials (coal, ore, etc.) at a distance of 20 to 100 meters, it is advisable to use inertial conveyors with a constant load pressure to the bottom of the chute [1]. These conveyors have considerable stability because the oscillation amplitude of the conveyor chute is constant.

Many domestic and foreign companies (KNAPP, SIAT, Blume, Librawerk, "Steklopak", "Siberian Machine-Building Company", "Potok-TM", "Project Invest") are engaged in research aimed at creating new-generation conveyors [1]. The widespread popularity of 
such studies is justified by the following factors: simplicity of construction, tightness, the possibility of combining the process of transportation with technological operations (sifting, drying, cooling), transportation of various materials of the construction industry (gypsum, sand, clinker, crushed stone, energy consumption), low chute wear compared to vibrating conveyors.

The inertial conveyors that transport goods due to the harmonious nature of the chute movement are particularly prominent. They have been used for transportation of various bulk and artificial loads, especially those where there is an undesirable mode of work with the tossing of material particles (heavy metal chips, hot wet sugar, etc.). Otherwise, when the inertial conveyor is in operation, there is no contact between the load and the rotating parts, such as in a screw conveyor.

On the surface of the inertial conveyor chute the load moves cyclically, that is, in one rotation of the crank chute performs a straightline movement, and the movement back and forth is carried out at different speeds. Due to this, the load moves on the surface of the chute with some average speed, on which the performance of the conveyor depends.

The kinematic characteristics of the chute movement, depending on the angle of crank rotation are shown in Fig. 1.

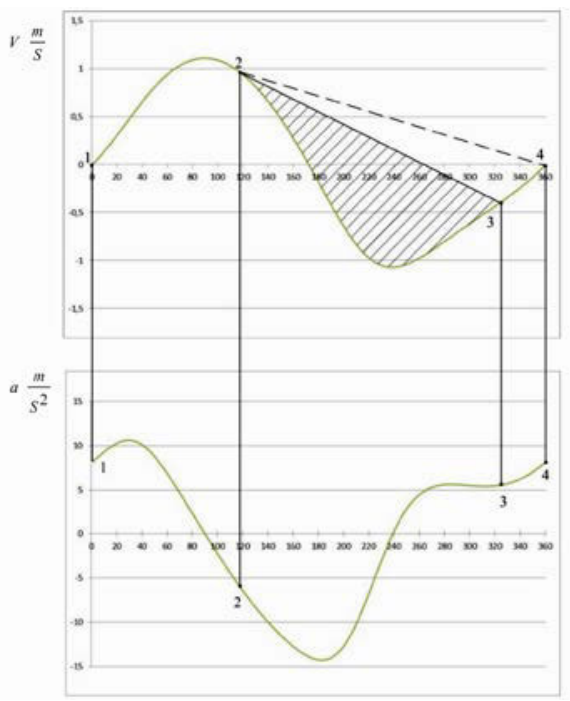


Fig. 1. The dependence of the velocity and acceleration of the inertial conveyor chute on the position and speed of the crank turn

Analysis of recent research and publications. In works[1, 2] the issues of kinematics and dynamics of inertial conveyors have been considered, which allow to judge its performance and energy costs for load movement and other operational characteristics.

The studies $[3,4]$ have been dedicated to the development of the construction and study of the inertial conveyor using linear asynchronous electric drive. These mechanisms have been used to transport wet sugar. Because, unlike vibration conveyors, they work without tossing loads, which leads to sugar segregation.

In scientific works [5,6], the results of studies of kinematic and dynamic parameters of inertial conveyors with elastic units have been presented. They can be used to select the optimal parameters when creating inertial conveyors, which are characterized by compactness and reduced material consumption and energy consumption.

The problems of studying the oscillations of dry friction systems, which arise in the study of the operation of inertial conveyors, are a typical example of the problems of nonlinear mechanics, which require special methods of analysis to be solved. These include: small parameter method, harmonic balance method, asymptotic methods and numerical methods [7-10].

The main disadvantage of inertial conveyors is the reduction of the velocity of the material being transported when the conveyor belt has been reversed due to friction. The conversion of dry friction into a binder allows the reduction of friction resistance in some selected direction, which is a prerequisite for creating a new generation of conveyor systems.

The main directions of the research are to solve the equations of bulk material particles motion on the surface of the chute, which performs transverse oscillations and the amplitude and frequency of which varies according to laws of different types. As a result, this action will optimize the construction of kinematic parameters of the inertial conveyor.

Among the publications on the issue of vibration displacement of solids and bulk materials, it is worth mentioning the works of Blehman I., Dzhanelidze G., Zayika P. [11,12]. 
The aim of the study formulation. The aim of the study is to establish the laws of bulk material particles motion on the surface, which performs a harmonic movement and to create on their basis constructions of high-performance inertial conveyors. In the framework of the work it is planned to develop a mathematical model of the movement of bulk material particles on the surface of the chute and determine the movement velocity of this material (conveyor performance), which make it possible to substantiate the rational construction and kinematic parameters of this vehicle. Also, get simple dependencies to determine the average movement velocity of the bulk material in a rectangular chute, which transversely oscillates in the plane of the bottom of the chute, perpendicular to the line of greatest slope.

The outline of the main research material. The construction of an inertial conveyor for bulk materials transportation has been elaborated in order to increase productivity and reduce the energy intensity of the bulk materials transportation process. The inertial conveyor consists of a chute, which performs reciprocating motion on a fixed basis and actuating the reciprocating movement of the chute. It should be mentioned that, the chute can perform not only a reciprocating motion, but also a movement in a direction that is perpendicular to the axis of the conveyor in a horizontal plane. It has been proposed to reduce the friction force between the load and the chute at a time when the speed of the chute relative to the load will be directed in the opposite direction [13].

The scheme of the proposed inertial conveyor is shown in Fig. 2

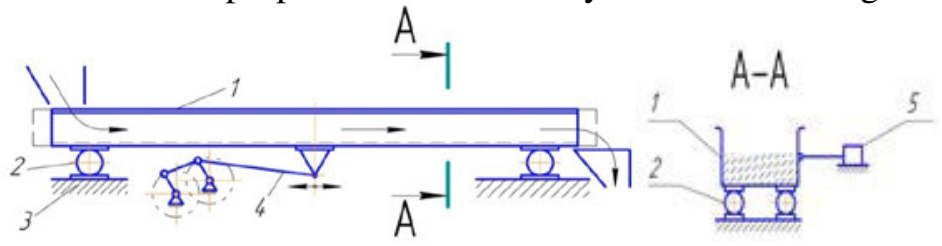

Fig. 2. Intertial conveyor

1 - chute; 2 - ball; 3 - base; 4 - reciprocating drive; 5 - vibrator

The inertial conveyor (Fig. 2) has a chute 1 that can move both along and across the axis of the conveyor on the balls 2 on a fixed base 3 , drive reciprocating motion 4 , vibrator 5 for the implementa- 
tion of transverse vibrations of the chute and a vibrator control that is not shown in the figures.

The principle of an inertial conveyor operation is as follows: chute 1 moves to the right by actuator 4 and the load on the chute begins to move with the chute. When the velocity of the chute 1 reaches the maximum value, the vibrator 5 is switched on and the chute begins to transverse. Thus, the friction force between the material and the chute will in most cases be directed in the transverse direction. Because the frequency of transverse oscillations is much greater than the frequency of longitudinal oscillations of the chute, but the friction force is always directed in the opposite direction from the vector of relative velocity of the material movement on the surface of the chute and now its longitudinal value will have a minimum value. Inertia, the material will move on the surface of the chute until the chute itself begins to move in the required direction. At this point in time, the vibrator shuts off and the cycle repeats.

Therefore, the studied inertial conveyor allows increasing productivity and improving the energy performance of the material transportation process [14].

The scheme and the general view of elaborated experimental setup are presented in Fig. 3 and Fig.4.

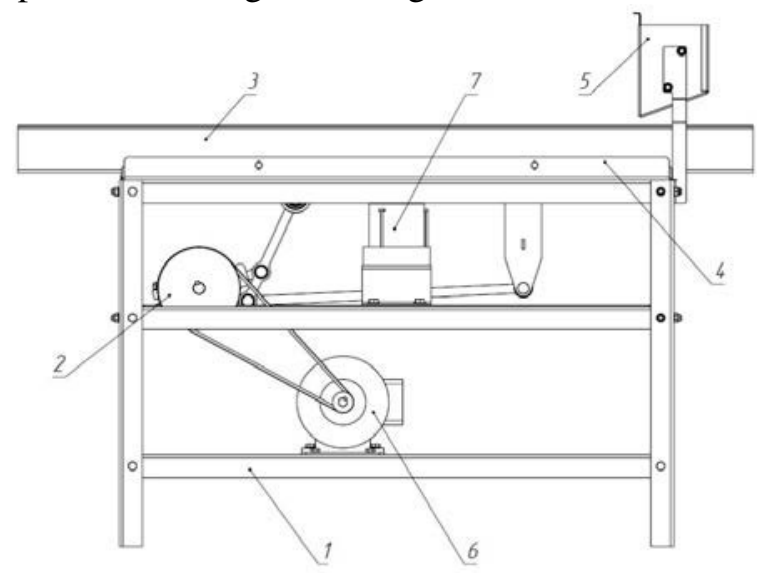

Fig. 3. The scheme of experimental setup:

1 - frame; 2 - chute drive; 3 - chute; 4 - guide of chute; 5 - material feed hopper; 6 - DC electric motor; 7 - vibrator. 


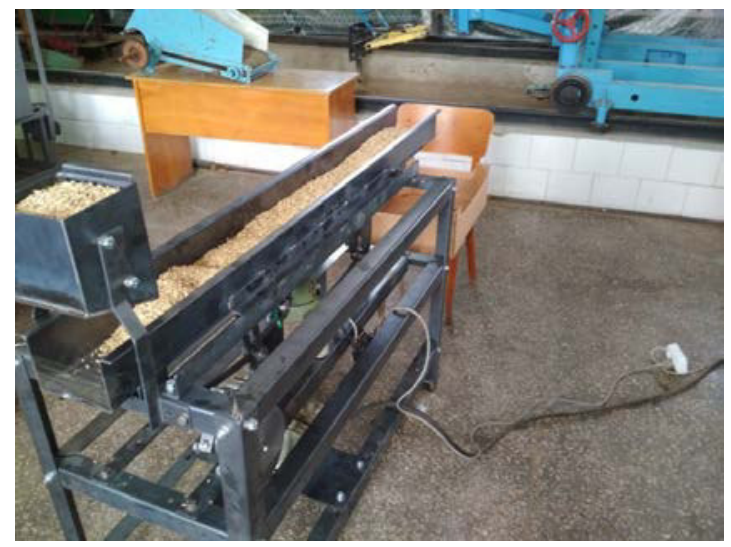

Fig. 4. General view of the elaborated inertial conveyor

The motion of the particle located on the surface of the groove of the inertial conveyor should be considered to determine the velocity of the bulk material [12].

The chute moves in a horizontal plane along the axis of the conveyor by law $S_{x}=S(t)$ and across the axis of the conveyor by law $S_{y}=A \sin \omega t$ (Fig. 5).

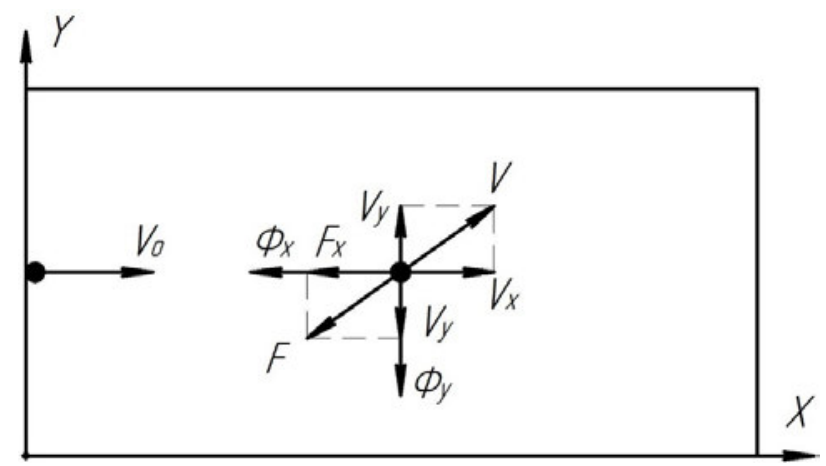

Fig. 5. The scheme of a particle motion of material on the surface of the chute

The force of gravity $\vec{P}$, the surface reaction $\vec{N}$, the friction force $\vec{F}$ and inertia forces $\vec{\Phi}_{X}, \vec{\Phi}_{y}$ are acting on the particle.

$$
\Phi_{x}=-m \ddot{S}_{x}
$$




$$
\Phi_{y}=-m \ddot{S}_{y} \sin \omega t
$$

Since the friction force directed in the opposite direction of the vector velocity point, then expand it into two parts $F_{X}$ and $F_{Y}$

$$
\begin{aligned}
& F_{x}=f_{N} \frac{V_{x}}{\sqrt{V_{x}^{2}+V_{Y}^{2}}} ; \\
& F_{y}=f_{N} \frac{V_{y}}{\sqrt{V_{x}^{2}+V_{y}^{2}}}
\end{aligned}
$$

where $V_{x}=x$ - the projection of the velocity vector of the particle on the axis $V_{y}=y,-$ the projection of the velocity vector of the particle on the axis $y$.

The motion law of the chute $S_{x}$ depends on the geometric and kinematic characteristics of the inertial conveyor drive.

The differential equations of a particle motion of the bulk material, in projections on the axis $x$ and $y$ will be the following

$$
\left\{\begin{array}{l}
\ddot{x}=-\ddot{S}_{x}-f g \sqrt{\frac{V_{x}}{V_{x}^{2}+V_{y}^{2}}} \\
\ddot{y}=A \omega^{2} \sin \omega t-f g \sqrt{\frac{V_{y}}{V_{x}^{2}+V_{y}^{2}}}
\end{array}\right.
$$

As the system of equations (5) is essentially nonlinear, so it cannot be integrated into quadratures and its periodic junction cannot be found in a closed form.

By solving the obtained system of equations by numerical method, the velocity of a particle motion of material on the surface of the chute under the given initial conditions of motion should be determined [15].

The obtained dependences of the coordinates and velocities of the particles of the bulk material on time, allow to determine the performance of an inertial conveyor with specified geometric and kinematic characteristics. The dependences of the velocity of a particle motion of the material on the horizontal surface on the frequency and amplitude of oscillations are given in Fig.6 [16]. 


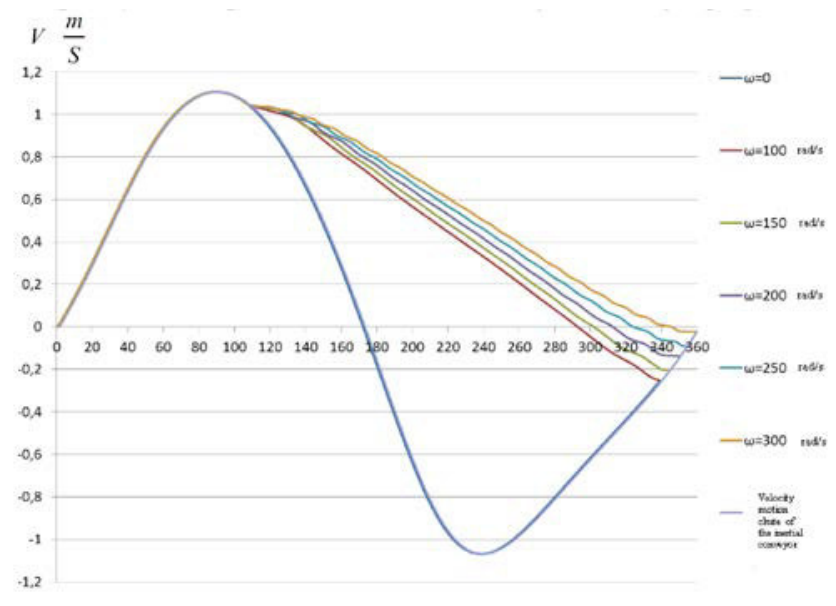

Fig. 6. The dependence of the velocity motion of the material on the chute surface of the inertial conveyor on the position of the crank and the vibration amplitude of the vibrator. ( $n=100 \mathrm{rev} / \mathrm{min}, \omega=150 \mathrm{rad} / \mathrm{s}$ )

The dependence of the load motion on the chute surface of the inertial conveyor on the frequency and the oscillation amplitude of the vibrator is given in Fig. 7 ( $n=100 \mathrm{rev} / \mathrm{min})$.

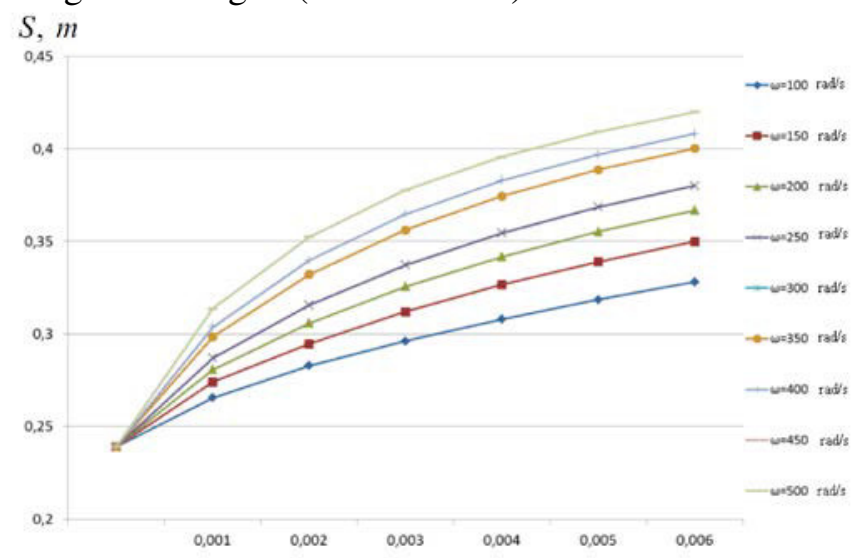

Fig. 7. The dependence of the load motion on the chute surface of the inertial conveyor on the frequency and the oscillation amplitude of the vibrator ( $n=100 \mathrm{rev} / \mathrm{min}$ ) 
The dependency response surface of load motion along the surface of the inertial conveyor chute from the frequency and oscillation amplitude of the vibrator is given in Fig.8.

The flow of bulk material in the chute, which is inclined at an angle $\alpha$ to the horizon, has been considered.

It has been assumed that the layer of bulk material with the height $\mathrm{h}$ is in a rectangular chute, which is located at an angle $\alpha$ to the horizon (Fig. 9) while $\alpha<\varphi$, where $\varphi$ - the sliding friction angle of the bulk material on the chute surface.

In the general case, to determine the velocity distribution of particles motion of bulk material in the chute, it is necessary to write the differential equations of motion of a continuous medium, continuity, barotropy and boundary state. Solving the differential equations obtained with partial derivatives, under certain boundary conditions, that is associated with considerable mathematical difficulties and it is possible to make them only under certain assumptions.

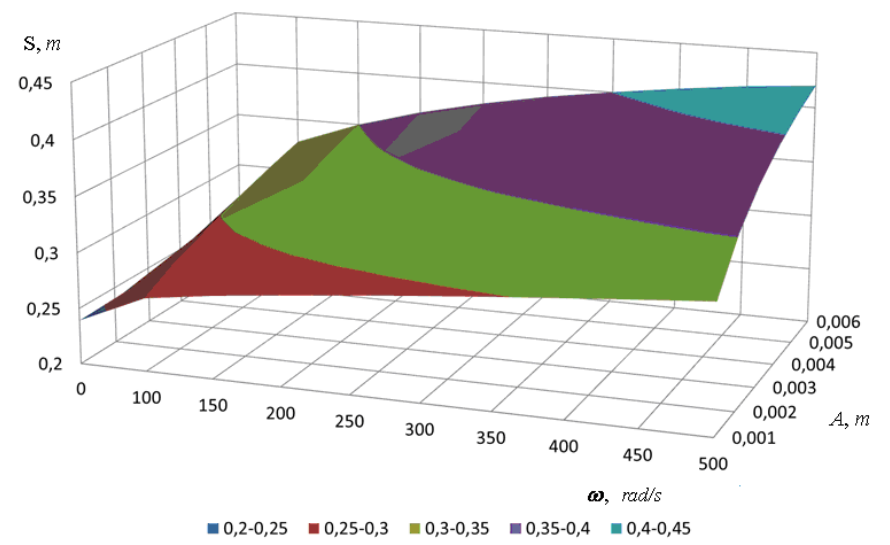

Fig. 8. The dependency response surface of load motion along the surface of the inertial conveyor chute from the frequency and oscillation amplitude of the vibrator at crank rotation speed $n=100 \mathrm{rev} / \mathrm{min}$

Consider the transverse harmonic oscillations of a high-intensity chute, where $A$ - oscillation amplitude, $\mathrm{m} ; \omega$ - oscillation frequency, $s^{-1}$. 


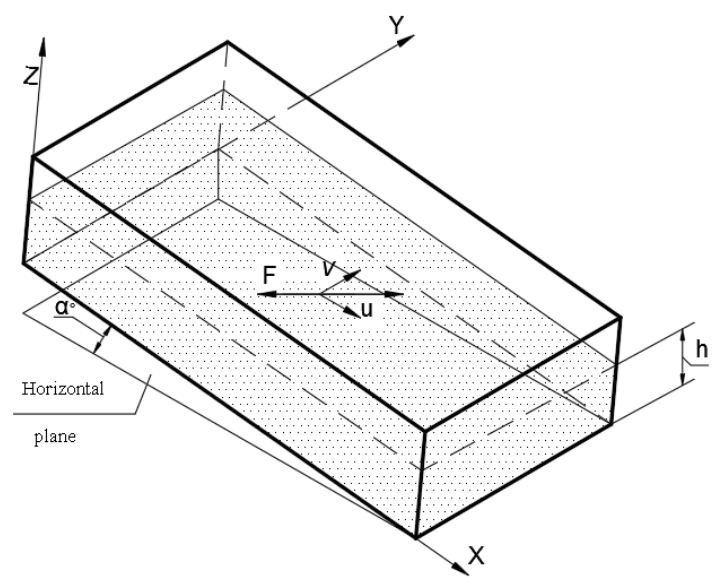

Fig. 9. The calculating scheme of the chute with a layer of bulk medium

Experimental studies $\left(A=0,001 \mathrm{~m}, \omega=140 \mathrm{~s}^{-1}\right)$ show that the bulk material does not change the rectangular shape, that is, during the period $T=\frac{2 \pi}{\omega}=0,045 \mathrm{~s}$ the particles of material do not have time to crumble.

In this case, the motion of the bulk medium in the chute can be considered as the motion of a solid body, which has the shape of a parallelepiped (Fig. 9).

Since the force of friction between the body and the bottom is always directed in the opposite direction of motion, its projections $\left(F_{x}\right.$ and $F_{y}$ ) on the coordinate axis will be

$$
\left\{\begin{array}{l}
F_{x}=f N_{z} \cdot \frac{u}{\sqrt{u^{2}+v^{2}}} \\
F_{y}=f N_{z} \cdot \frac{u}{\sqrt{u^{2}+v^{2}}}
\end{array}\right.
$$

where $u=\dot{x}$ - velocity of the body, $\mathrm{m} / \mathrm{s}, v$ - the velocity of the chute in the transverse direction, $\mathrm{m} / \mathrm{s}$.

As $N_{\mathrm{z}}=\mathrm{mg} \cos \alpha$, so

$$
F_{x}=f m g \cos \alpha \cdot \frac{u}{\sqrt{u^{2}+v^{2}}}
$$


The differential equation of motion of this body in the projection on the axis $x$

$$
m \ddot{x}=m g \sin \alpha-f m g \sin \alpha \cdot \frac{u}{\sqrt{u^{2}+v^{2}}} .
$$

In our case, the chute performs transverse oscillations by law $y=A \sin \omega t$

The projection of the chute velocity on the $y$-axis will be $v=\dot{y}=A \omega \cos \omega t$.

Then the differential equation of motion of the material on the surface of the chute will look like

$$
m \ddot{x}=m g \sin \alpha-f m g \cos \alpha \cdot \frac{\dot{x}}{\sqrt{\dot{x}^{2}+(A \omega \cos \omega t)^{2}}}
$$

The resulting equation does not integrate into quadratures and its solution cannot be found in general form, but it is possible to get an approximate solution [7].

Dimensionless quantities

$$
\tau=\omega t ; \xi=\frac{x}{A} ; \dot{x}=\frac{d x}{d t}=\frac{d(\xi A) \cdot \omega}{d \tau}=A \omega \xi^{\prime},
$$

where $\xi^{\prime}$ derivative of $\xi$ in dimensionless time $\tau$

$$
\ddot{x}=\frac{d(\ddot{x})}{d t}=\frac{d\left(A \omega \xi^{\prime}\right) \cdot \omega}{d \tau}=A \omega^{2} \frac{d \xi}{d \tau} .
$$

Equation (7) takes the form

$$
A \omega^{2} \frac{d \xi^{\prime}}{d \tau}=g \sin \alpha-f g \cos \alpha \cdot \frac{A \omega \xi^{\prime}}{\sqrt{\left(A \omega \xi^{\prime}\right)^{2}+A^{2} \omega^{2} \cos _{2} \tau}}
$$

The notation is entered

$$
\mu=\frac{f g \cos \alpha}{A \omega^{2}} ; \quad y=\frac{g \sin \alpha}{f \cos \alpha}=\frac{\tan \alpha}{f} .
$$

Finally (6) in dimensionless form

$$
\frac{d \xi}{d t}=\mu\left(y-\frac{\xi^{\prime}}{\sqrt{\xi^{\prime 2}+\cos 2 \tau}}\right) \text {. }
$$

Because in our case 


$$
A \omega^{2}>>\frac{g t}{\cos \alpha} .
$$

Therefore, $\mu$-value in this equation is a small parameter and the averaging method to solve the equation can be used. It should be assumed that velocity is constant over a period of oscillation $v$ [7].

$$
v=\left\langle\xi^{\prime}\right\rangle=\frac{1}{2 \pi} \int_{0}^{2 \pi} \xi^{\prime}(\tau) d \tau
$$

The expression under the sign of the radical from equation has been transformed as (9)

$$
\begin{aligned}
& \sqrt{\xi^{\prime 2}+\cos ^{2} \tau}=\sqrt{\xi^{\prime 2}+\left(1-\sin ^{2} \tau\right.}=\sqrt{v^{2}+1-\sin ^{2} \tau}= \\
& =\frac{1}{k} \sqrt{1-k^{2} \sin ^{2} \tau},
\end{aligned}
$$

where, $\int_{0}^{\frac{\pi}{2}} \frac{1}{\sqrt{1-k^{2} \cos ^{2} \tau}} \cdot d \tau=K(v)$ - a complete elliptic integral of the first kind whose modulus is equal to $k$.

Finally it will be

$$
\begin{aligned}
& \frac{d v}{d \tau}=\mu\left(\gamma-\frac{2 v}{\pi} \cdot k(v) \cdot K(k)\right) ; \\
& \varphi(v)=\frac{2 v}{\pi} k(v) \cdot K(k) .
\end{aligned}
$$

Tables for finding elliptic integrals [17] can be used to determine the value of a function $\varphi(\gamma)(12)$, which has been shown in Table 1 and in Fig. 10

Table1

The value of function dependency $\varphi(\gamma)$ versus the dimensionless velocity $v$

\begin{tabular}{c|c|c|c|c|c|c|c|c|c|c|c}
\hline$\square$ & 0,0 & 0,1 & 0,2 & 0,3 & 0,4 & 0,5 & 0,6 & 0,7 & 0,8 & 0,9 & 1,0 \\
\hline$k(v)$ & 1 & 0,995 & 0,981 & 0,958 & 0,928 & 0,894 & 0,857 & 0,819 & 0,781 & 0,743 & 0,707 \\
\hline$K(\mathrm{k})$ & $\infty$ & 3,7 & 3,04 & 2,67 & 2,43 & 2,26 & 2,13 & 2,035 & 1,96 & 1,9 & 1,85 \\
\hline$\varphi(v)$ & 0 & 0,234 & 0,379 & 0,488 & 0,575 & 0,643 & 0,698 & 0,743 & 0,780 & 0,809 & 0,834 \\
\hline
\end{tabular}

\begin{tabular}{c|c|c|c|c|c|c|c|c|c|c|c}
\hline$v$ & 1,0 & 1,1 & 1,2 & 1,3 & 1,4 & 1,5 & 1,6 & 1,7 & 1,8 & 1,9 & 2,0 \\
\hline$k(v)$ & 0,707 & 0,673 & 0,640 & 0,610 & 0,581 & 0,554 & 0,530 & 0,507 & 0,486 & 0,466 & 0,447 \\
\hline$K(k)$ & 1,85 & 1,82 & 1,78 & 1,76 & 1,74 & 1,72 & 1,70 & 1,69 & 1,68 & 1,67 & 1,66 \\
\hline$\varphi(v)$ & 0,834 & 0,855 & 0,870 & 0,888 & 0,901 & 0,911 & 0,918 & 0,927 & 0,934 & 0,940 & 0,945 \\
\hline
\end{tabular}


Continuation of table. 1

\begin{tabular}{c|l|l|l|l|l|l|l|l|l|l|l}
\hline$v$ & 2,0 & 2,1 & 2,2 & 2,3 & 2,4 & 2,5 & 2,6 & 2,7 & 2,8 & 2,9 & 3,0 \\
\hline$k(v)$ & 0,447 & 0,430 & 0,414 & 0,399 & 0,385 & 0,371 & 0,359 & 0,347 & 0,336 & 0,326 & 0,316 \\
\hline$K(k)$ & 1,66 & 1,65 & 1,645 & 1,64 & 1,63 & 1,63 & 1,625 & 1,62 & 1,62 & 1,615 & 1,61 \\
\hline$\varphi(v)$ & 0,945 & 0,950 & 0,953 & 0,958 & 0,960 & 0,964 & 0,966 & 0,968 & 0,970 & 0,972 & 0,974 \\
\hline
\end{tabular}

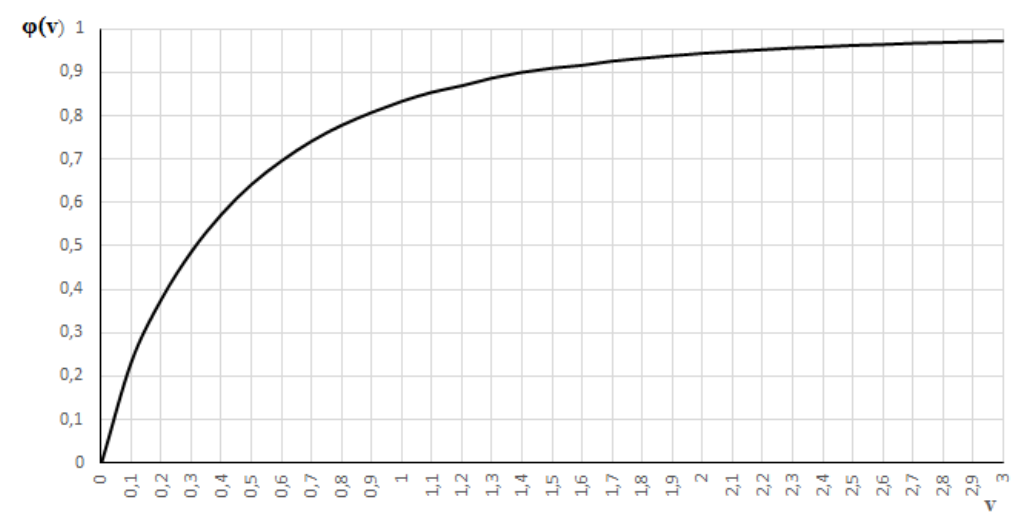

Fig. 10. The Graph of function dependency $\varphi(\gamma)$ versus the dimensionless velocity $v$

From the form of equation (12) and table 1 it is clear that when $\gamma<1$ there is a steady-state regime in the system, while $\gamma=\frac{\tan \alpha}{\tan \varphi}>1$, $a>1$, so the motion will occur in the absence of transverse oscillations and will be equally accelerated, because the projection of the gravitational force on the $x$-axis will be greater than the friction force.

Fixed values of the average velocity $v$ have been found from the equation

$$
\frac{2 v}{\pi} \cdot k(v) \cdot K(k)=\gamma
$$

Given the values of $\gamma=\varphi(\gamma)$ in Table 1 , the dimensionless average velocity of the bulk material in steady motion has been determined.

Table 2 shows the dimensionless average velocity $v$ of the bulk material motion on the chute surface, which performs transverse oscillations, from the inclination chute angle $\alpha$ to the surface and the friction coefficient $f$, and in Fig. 11. the response surface has been shown. 
Table 2

The dependence value of the average velocity of the bulk material on the chute surface, from the inclination chute angle $\alpha$ to the surface and the friction coefficient $f$

\begin{tabular}{|c|c|c|c|c|c|}
\hline$\alpha^{\circ}$ & 10 & 12 & 14 & 16 & 18 \\
\hline 0,35 & 0,318 & 0,447 & 0,631 & 0,94 & 1,7 \\
\hline 0,4 & 0,257 & 0,348 & 0,471 & 0,642 & 0,912 \\
\hline 0,45 & 0,212 & 0,285 & 0,376 & 0,491 & 0,653 \\
\hline 0,5 & 0,168 & 0,242 & 0,313 & 0,4 & 0,505 \\
\hline
\end{tabular}

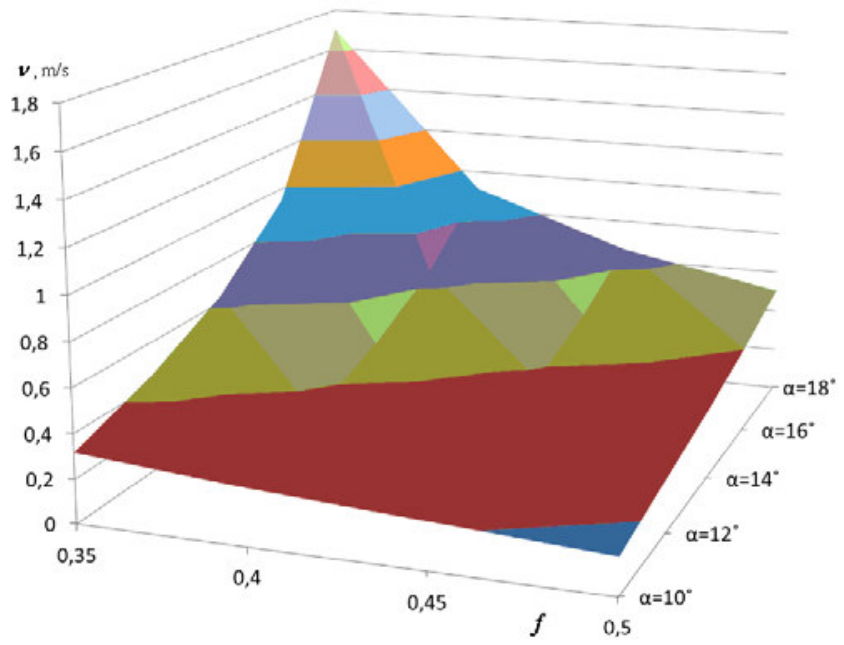

Fig. 11. The dependency response surface of the average velocity motion of the bulk material on the chute surface, which performs transverse oscillations, from the inclination chute angle $\alpha$ to the surface and the friction coefficient $f$

The dimensional average velocity $U$ has been determined from equation

$$
U=A \omega v \text {. }
$$

Table 3 shows the results of theoretical and experimental studies of the bulk material motion on the chute surface (the material is barley, the coefficient of friction $f=0,5$, oscillation amplitude $A=0,001 \mathrm{~m}$, oscillation frequency $\omega=140 \mathrm{~s}^{-1}$ ). 
Table 3

The results of theoretical and experimental studies of the bulk material motion on the chute surface

\begin{tabular}{l|l|l|l|l}
\hline$\alpha^{\circ}$ & 10 & 12 & 14 & 16 \\
\hline$U_{\mathrm{T},} \mathrm{m} / \mathrm{s}$ & 0,023 & 0,034 & 0,044 & 0,056 \\
\hline$U_{\mathrm{E}}, \mathrm{m} / \mathrm{s}$ & 0,02 & 0,029 & 0,035 & 0,054 \\
\hline$\delta, \%$ & 15 & 17,2 & 12,8 & 7 \\
\hline
\end{tabular}

A comparative analysis of the theoretical results confirmed their satisfactory agreement with the experimental data, in contrast to [2], where the average values of the velocity motion of a particle have been significantly different from the experimental data [18].

\section{Conclusions}

As a result of conducted theoretical researches, it has been found that by providing the chute an inertial conveyor of transverse oscillations during periods of time when the load slides on the surface of the chute, the performance of the vehicle is significantly improved. Thus, at the frequency of transverse oscillations $\omega=500 \mathrm{rad} / \mathrm{s}$ and the amplitude of oscillations $A=0.005 \mathrm{~m}$, the productivity of the conveyor is increased by $20 \%$.

Currently, the program and methodology of additional experimental studies have been elaborated. Due to given actions the adjustments will be made to the construction of the inertial conveyor and rational geometric and kinematic parameters will be proposed, both as the chute motion driver, as well as the frequency and amplitude of the chute transverse oscillations, which ensure an efficient process of transporting bulk materials through this device.

On the consequences of provided studies of the bulk material motion in a rectangular chute, which performs transverse oscillations with high intensity, the dependence of the average velocity of the material on the inclination chute angle to the horizon, the sliding friction coefficient and the oscillation parameters have been obtained.

These dependencies can be used in engineering calculations of the motion of flowing bulk material while solving specific practical problems.

\section{References}

1. Timofeev G.A., Kuzenkov V.V. New approaches to the study of the dynamics of inertial conveyors / G.A. Timofeev, V.V. Kuzenkov // "News of higher educational institutions." - Moscow: N.E. Bauman MSTU., 2013 - P. 24 - 30. 
2. Dyakonova V.Ya. Dynamic and kinematic motion parameters of the inertial conveyor chute. Collection of Works "Advanced Materials, Technologies, Structures, Economics". - Krasnoyarsk, 2004. - P. 203-213.

3. Aipov R.S., Shagargazin A.S. Swing conveyor based on a linear electric drive for transporting and drying grain: "Achievements of agricultural science - production. Materials of 110 scientific-practical conference for teachers, employees and graduate students of the university. - Ufa: BGAU, 2004.- 160p.

4. Akchurin S.V. Inertial conveyor of wet sugar with a link for preliminary acceleration of a linear asynchronous electric drive: Thesis of PhD. tech. Sciences: 05.20.02. St. Petersburg. Pushkin: RSL, 2013.

5. Dyakonova V.Ya., Kalinovskaya T.G., Kosolapova S.A., Dyakonov M.N. The statement of the problem of studying the drive of an inertial conveyor with drafts of variable stiffness // News of Higher Education Mountain Journal. - 2014. - No. 1. - P. 118-122.

6. Dyakonova V.Ya., Kuzmenko V.A., Bratukhina N.A. Experimental studies of inertial conveyor with flexible traction. Collection of scientific articles "Scientific and technological progress." - Krasnoyarsk, 1994.

7. Andronov V.V., Zhuravlev F.V. Dry friction in problems of mechanics. - M. Izhevsk: Research Center "Regular and Chaotic Dynamics", Institute for Computer Research, 2010. - $184 \mathrm{p}$.

8. Biderman V.L. Theory of mechanical vibrations. - M.: Higher School, 1980. - 408 p. $510 \mathrm{p}$.

9. The vibration in technology. Handbook in 6 volumes. Vol. 4. M.: Engineering, 1981. -

10. Loveveykin V. S. Application of phase-frequency methods of influence on the parameters of the forced power to minimize the dynamic coefficients of vibration systems with dry friction / V. S. Loveveykin, Yu. V. Chovnyuk, K. I. Pochka // The Bulletin of National University "Lviv Polytechnic". Dynamics, durability and design of machines and appliances. - 2013. № 759. - P. 48-54.

11. Blehman I. I., Ddhanelidze G.Yu. Vibration movement. - M.: Science, 1964. - 412 p.

12. Zaika P. M. Vibration Movement of Solids and Bulk Bodies in Agricultural Machines: Pract. Allowance. - Kiev: USHA Publishing House. - 199 p.

13. Rogatinsky R. M., Serilko L. S., Serilko D. L., Kozachuk R. I. Inertial conveyor / utility model patent No. 111199. Bul. No. 21, 2016.

14. Serilko L. S. New Constructions of Inertial Conveyors / LS Serilko, DL Serilko // Proceedings of the XIX Scientific Conference of Ternopil Ivan Puluj National Technical University: Abstracts. - Ternopil, 2016. - P. 73.

15. Serilko L. S. Investigation of bulk material motion on the chute of an inertial conveyor / LS Serilko, DL Serilko, R.I. Kozachuk // Materials of the XX Scientific Conference of Ternopil Ivan Puluj National Technical University: Abstracts. - Ternopil, 2017. - P. 49-50.

16. Rogatinsky R. M., Serilko L. S., Sasyuk Z. K., Serilko D. L. Investigation of the dynamics of inertial conveyors // Vibrations in Engineering and Technology, 2018. №2 (89). P.41-48.

17. Yanke E., Emde F., Lesh F. Special functions. M.: Nauka, 1968, 344 p.

18. Serilko L. S., Sasyuk Z. K., Shchuryk V. A., Serilko D. L. Determination of the average velocity of bulk material in a rectangular chute, which carries out transverse oscillations // Vibrations in Engineering and Technology, 2018. №3 (90) P.99-104. 